\title{
Les gestes postérieurs de décompression
}

\section{Posterior decompression of the vertebral canal}

\author{
M. Perrin
}

Le Point Médical, Rond Point de la Nation, F-21000 Dijon Cedex

\begin{abstract}
The single indication for laminectomy at present is neurosurgical and it is widespread epiduritis without any compressive bony or unstable lesion of the spine.
\end{abstract}

Key words : Bone metastases - Spine

Résumé. La seule indication actuelle de la laminectomie est neuro-chirurgicale: c'est l'épidurite étendue sans lésion osseuse comprimant le rachis ou le rendant instable.

Mots-clés : Métastases osseuses Rachis

La laminectomie était autrefois la technique de routine pour traiter les paraplégies. Quelle que soit l'origine de la compression, sa situation, son mécanisme, le risque médullaire était la seule justification de ce geste urgent de décompression. Il faut dire que l'imagerie médicale ne s'est développée que depuis 5 ans et qu'auparavant la myélographie ne pouvait faire que préciser le niveau de la compression.

La laminectomie est déstabilisante. Le schéma biomécanique (fig. 1) des trois colonnes, de l'amortisseur discal antérieur, des articulations postérieures

Code Méary : 0499.1 et du frein à l'écrasement discal représenté par le ligament interépineux et les ligaments jaunes, montre bien pourquoi.

Un autre type de geste décompressif est possible par voie postérieure, c'est la neurolyse radiculaire (fig. 2). Elle a bien peu de place dans ce contexte, mis à part en colonne lombaire, lorsqu'une radiculalgie a été mal calmée par la radiothérapie et qu'elle devient rebelle aux antalgiques. Cette indication est évoquée mais, en pratique, n'est pas réalisée.

Les lésions postérieures sont de trois types : métastase épidurale isolée ou associée à des lésions osseuses, métastase osseuse de l'arc postérieur limitée, métastase postérieure associée à une atteinte des massifs articulaires et du mur postérieur ou du corps, ces atteintes globales étant de loin les plus fréquentes.

Les épidurites isolées sont traitées par radiothérapie si elles ne dépassent pas trois étages ; au delà de cette importance la laminectomie est justifiée. Les atteintes postérieures limitées sont traitées par radiothérapie. Celles qui compromettent la stabilité sont traitées

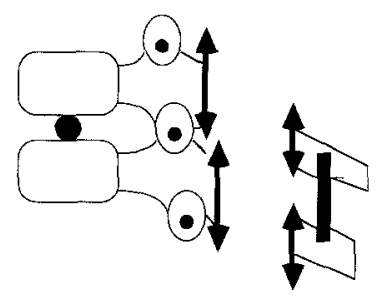

Fig. 1. La laminectomie déstabilisante par voie postérieure avec un matériel d'ostéosynthèse efficace. L'ouverture du canal rachidien n'est pas systématique. Elle est réalisée pour lever une compression postérieure vue sur le bilan radiologique pré-opératoire et qui n'a aucune chance de se modifier, à la différence des compressions antérieures, avec la mise en place d'un appareillage même, et surtout, s'il est en distraction. Il est certain que l'intervention est avantageusement réalisée avant la survenue d'une paraplégie. Toutefois, devant une paraplégie incomplète, si l'on ne possède pas le matériel ou la technicité suffisante pour stabiliser de façon convenable le segment rachidien considéré, l'attitude qui consiste à réaliser en urgence une laminectomie décompressive permettant d'attendre le geste stabilisateur est très discutable.

La seule indication actuelle de la laminectomie est neuro-chirurgicale : c'est l'épidurite étendue sans lésion osseuse comprimant le rachis ou le rendant instable.

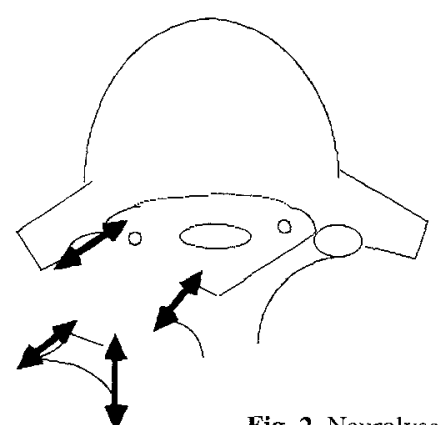

Fig. 2. Neurolyse radiculaire 